\title{
Erratum: Alleviation of the Fermion-Sign Problem by Optimization of Many-Body Wave Functions [Phys. Rev. Lett. 98, 110201 (2007)]
}

\author{
C. J. Umrigar, Julien Toulouse, Claudia Filippi, S. Sorella, and R. G. Hennig \\ (Received 19 September 2007; published 24 October 2007)
}

In our original Letter we omitted acknowledging the following sources of support.

This work was supported in part by DOE Grant No. DE-FG02-99ER45795, NSF Grant No. EAR 0530813 and No. EAR 0703226, and the Marie Curie Outgoing International Fellowship (No. 039750-QMC-DFT). Computational resources were also provided by the Cornell Nanoscale Facility. 